\title{
Molecular outflows in the young open cluster IC $348^{1}$
}

\author{
Jochen Eislöffel, Dirk Froebrich \\ Thüringer Landessternwarte Tautenburg, Sternwarte 5, D-07778 Tautenburg, Germany \\ Thomas Stanke \\ Max-Planck-Institut für Radioastronomie, Auf dem Hügel 69, D-53121 Bonn, Germany \\ Mark J. McCaughrean \\ Astrophysikalisches Institut Potsdam, An der Sternwarte 16, D-14482 Potsdam, Germany
}

\begin{abstract}
We present a wide-field survey of the young open cluster IC 348 for molecular $\mathrm{H}_{2}$ outflows. Outflow activity is only found at its south-western limit, where a new subcluster of embedded sources is in an early phase of its formation. If the IC 348 cluster had been built up by such subclusters forming at different times, this could explain the large age-spread that Herbig (1998) found for the IC 348 member stars. In addition to several compact groups of $\mathrm{H}_{2}$ knots, our survey reveals a large north-south oriented outflow, and we identify the newly discovered far-infrared and mm-object IC $348 \mathrm{MMS}$ as its source. New deep images in the 1-0 S(1) line of molecular hydrogen trace the HH 211 jet and counterjet as highly-collimated chains of knots, resembling the interferometric $\mathrm{CO}$ and $\mathrm{SiO}$ jets. This jet system appears rotated counter-clockwise by about $3^{\circ}$ with respect to the prominent $\mathrm{H}_{2}$ bow shocks. Furthermore, we resolve $\mathrm{HH}$ 211-mm as a double point-like source in the mm-continuum.
\end{abstract}

Subject headings: Shock waves - ISM: jets and outflows - ISM: kinematics and dynamics - ISM: molecules - stars: mass-loss

\section{Introduction}

The early stages of star formation are acompanied by powerful bipolar outflows. The investigation of such outflows helps us to better understand the star formation process as a whole. On the other hand, the flows are also useful as pointers to their sources, which in the earliest phases of their formation are deeply embedded in their parental clouds and not visible at optical or near-

\footnotetext{
${ }^{1}$ Based on observations taken at the German-Spanish Astronomical Centre, Calar Alto, operated by the MaxPlanck-Institute for Astronomy, Heidelberg, jointly with the Spanish National Commission for Astronomy and on observations with ISO, an ESA project with instruments funded by ESA Member States (especially the PI countries: France, Germany, the Netherlands and the United Kingdom) and with the participation of ISAS and NASA.
}

infrared wavelengths. Once the flows start breaking out of these dense cores into regions of lower extinction, the shock excited gas produced on their way through the ambient medium becomes observable in the near-infrared. An excellent tracer for these flows then is the emission of molecular hydrogen in its $1-0 \mathrm{~S}(1)$ line at $2.122 \mu \mathrm{m}$.

IC 348 is a young open cluster in the Perseus dark cloud. Several hundred cluster members have been found in the optical (Herbig 1998), in the near-infrared (Lada and Lada 1995; Luhman et al. 1998; Najita et al. 2000) and in X-rays (Preibisch et al. 1996; Preibisch and Zinnecker 2001). Herbig (1998) investigated the age of the cluster and found a mean age of $1.3 \mathrm{Myr}$, with a surprisingly large spread around this value of 0.7 to $12 \mathrm{Myr}$. This large spread is quite unusual for 
such a young cluster, and it may point to an unusual formation history. Since Herbig derived the ages only for optically visible stars, his lower limit may also imply that star formation is still going on in this cluster: There may be even younger objects, which cannot be detected in the optical because they are still too deeply embedded.

Indeed, Strom et al. (1974) made an IR-map of IC 348, and found a bright IR source (IC 348-IR) in a small red reflection nebula, which was later studied at high spatial resolution by Boulard et al. (1995). In its neighbourhood Mc Caughrean et al. (1994) discovered a highly-collimated molecular outflow, HH 211, in the south-western part of the cluster. In this region Bachiller, Guilloteau \& Kahane (1987) had already found several large clumps in ammonia and CO. The HH 211 flow has subsequently been studied in detail in highresolution interferometric observations in $\mathrm{CO}$ by Gueth and Guilloteau (1999) and in SiO by Chandler and Richer (2001). It source is suspected to be a Class 0 object, a protostar in its earliest stage of formation going through its main accretion phase.

On the $\mathrm{K}^{\prime}$ mosaic of Mc Caughrean et al. (1994) a chain of knots was seen north of HH 211, that could be part of another outflow. This prompted us to carry out a survey in molecular hydrogen of the entire IC 348 cluster to search for outflows and identify their sources, as a possibly so far unknown very young population in this cluster.

In the following we first describe our observations and data reduction in Sect. 2. We then present our new deep imaging of the HH 211 flow in Sect.3, and continue with the newly found $\mathrm{H}_{2}$ knots and flows in Sect. 4. Sect. 5 is devoted to a discussion of the possible outflow sources and the recent star formation in IC 348.

\section{Observations and Data Reduction}

\subsection{Near-infrared data}

Our near-infrared data were taken during several observing runs at the 3.5-m telescope on Calar Alto. In November 1995 we observed the region of the HH 211 outflow with the MAGIC infrared camera (Herbst et al. 1993). The high resolution optics yielded a scale of 0 .'32 per pixel. Narrowband filters centered on the 1-0 S(1) line of molec- ular hydrogen at $2.122 \mu \mathrm{m}$ and on the nearby continuum at $2.140 \mu \mathrm{m}$ were used. We observed a small mosaic with a per pixel integration time of $1740 \mathrm{~s}$. The seeing was about 0.9 . These data were not flux calibrated, because the weather conditions were not photometric.

In September 1997 the whole IC 348 cluster was observed under photometric weather conditions. This time, we used MAGIC with its wide field optic with a scale of 0.'81 per pixel. Again the narrow-band filter centered at $2.122 \mu \mathrm{m}$ with a FWHM of $0.019 \mu \mathrm{m}$ was used. The per-pixel integration time was $180 \mathrm{~s}$, and the seeing was about 1 .'2. Deeper mosaics in the $\mathrm{H}_{2}$ 1-0 $\mathrm{S}(1)$ line and in the continuum were taken of the region of the newly discovered outflows. They have integration times of $620 \mathrm{~s}$ and $520 \mathrm{~s}$ per pixel, and have been photometrically calibrated using the standards HD 18881, HD 203856, HD 3029 and HD 40335. In September 1997 a $13^{\prime} \times 13^{\prime}$ area around HH 211 was also observed with the Omega Prime camera (Bizenberger et al. 1998), at lower sensitivity, but covering an area extending further south than the MAGIC data.

In December 2000 we used again the Omega Prime camera with a scale of 0. .'396 and a field of view of $6 ! 8 \times 6 ! 8$. The weather conditions were photometric and the seeing about 1..5. Again we used a $\mathrm{H}_{2} 1-0 \mathrm{~S}(1)$ narrow-band filter at $2.122 \mu \mathrm{m}$, with an integration time per pixel of $810 \mathrm{~s}$. For flux calibration we observed the standard star FS13.

Flatfielding of the images was done using lamp on/off flats. For sky subtraction and co-addition we used the DIMSUM package in IRAF. The single images were co-centred onto a large reference frame using all detectable stars in the field, to ensure a high astrometric accuracy. Standard star images were reduced in the same way. Photometric errors are on the order of 0.05 mag. We used HST guide stars to determine the astrometric solution and reached a positional accuracy of about 0 . 5 . Emission features were identified by comparing the images taken in $\mathrm{H}_{2}$ and in the continuum in all cases.

\subsection{Far-infrared and $\mathrm{mm}$ data}

Small maps of the HH 211 region were taken with the PHOT instrument onboard the ISO satellite in its PHT22 mode and the C200 detector 


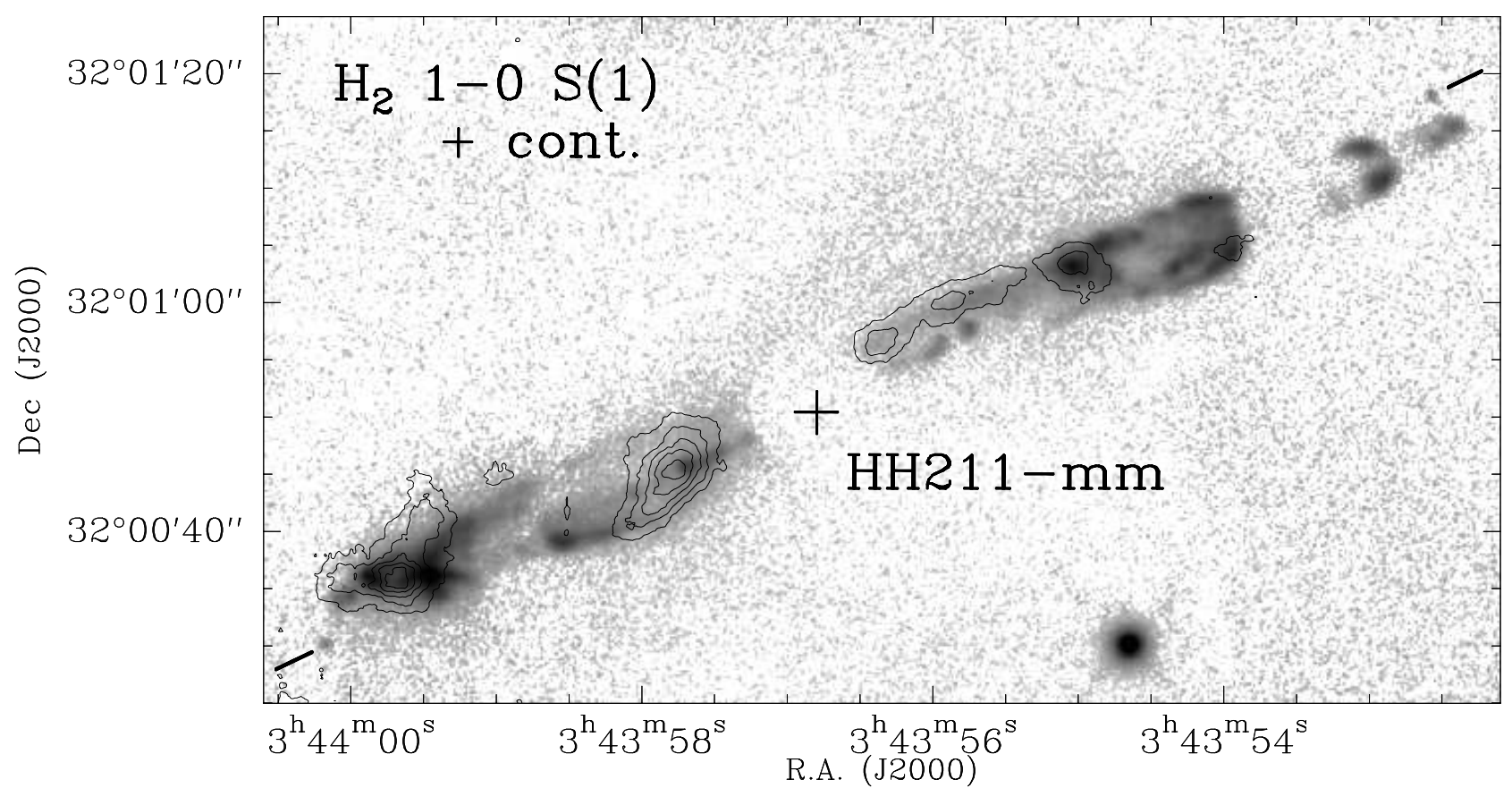

Fig. 1.- Grey-scale plot of the HH 211 outflow in the $1-0 \mathrm{~S}(1)$ line of molecular hydrogen at $2.122 \mu \mathrm{m}$, with logarithmic intensities. The contours show the continuum at $2.140 \mu \mathrm{m}$. A cross marks the position of the outflow source HH 211-mm. The orientation of the highly-collimated knotty jet and counterjet seen in $\mathrm{H}_{2}$ is marked by solid lines on both ends. This jet system is rotated counter-clockwise by about $3^{\circ}$ with respect to the system of the prominent $\mathrm{H}_{2}$ bows. The two point-like features next to the solid lines are likely background stars.

by stepping the instrument by half a detector pixel. This way, $7 \times 3$ mosaics with a pixel size of $45^{\prime \prime} \times 90^{\prime \prime}$, and a total field of view of $315^{\prime \prime} \times 270^{\prime \prime}$ were obtained. The data were reduced with the ISOPHOT Interactive Analysis (PIA V9.1) software. Fluxes of the sources were measured by attributing pixels manually to "object" or "background", then summing up the object flux and subtracting the background.

Our mm-continuum observations were obtained in March 1999 at the IRAM 30-m antenna using the 37-channel bolometer array MAMBO (Kreysa et al. 1998) at a wavelength of $1.2 \mathrm{~mm}$. An area of about $36 \square^{\prime}$ was covered. The data were reduced with MOPSI (software package developed by R. Zylka, pers. comm.) following standard procedures. After an initial despiking and low-order baseline subtraction the data were corrected for atmospheric extinction and flux calibrated. Then correlated sky brightness variations ("sky-noise") were removed in an iterative manner, using the result of previous runs as an input source model to improve the performance of the sky-noise removal. After further despiking and residual baseline subtraction the data were restored from dual beam to single beam and finally converted into a map.

\section{The HH 211 outflow}

In Fig. 1 we present a new image of $\mathrm{HH} 211$ in the $1-0 \mathrm{~S}(1)$ line of molecular hydrogen at $2.122 \mu \mathrm{m}$. This deeper image reveals some important details, which were not visible on the original data by Mc Caughrean et al. (1994). Most interestingly, we clearly see highly-collimated chains of knots in the jet and the counterjet. They are tracing the high-velocity molecular flows, which are seen in the interferometric observations in CO by Gueth and Guilloteau (1999) and in $\mathrm{SiO}$ by Chandler and Richer (2001). This chain of knots is running roughly through the middle of the rim-brightened cavity-like structure. A line drawn through the knots marking the highvelocity jet (indicated by the solid lines at both ends of the jet in Fig. 1) does not, however, ex- 
actly lead through the tips of the prominent $\mathrm{H}_{2}$ bows. Apparently this jet system is rotated about $3^{\circ}$ counter-clockwise with respect to the broader outflow lobes. This misalignment, which is also seen in some other jets, may be indicative of precession in the HH 211 flow. Indeed, we will show in Sect. 5 that the HH 211 source may be a binary.

Comparison with continuum images also shows that part of the rim-brightened cavity - especially the part close to the source and almost parallel to the western jet - is not $\mathrm{H}_{2}$, but instead continuum emission (see the contour plot in Fig. 1, which shows the continuum at $2.140 \mu \mathrm{m}$ ). Here we are probably seeing scattered light from the source that escapes from the dense shielding envelope through the jet channels, thus opening up the possibility of indirectly obtaining a spectrum of HH 211-mm.

\section{New outflows and $\mathbf{H}_{2}$ emission features in IC 348}

Our full $\mathrm{H}_{2}$ survey covers a $390 \square^{\prime}$ region centered on the young cluster IC 348 (between R.A.(J2000) $3^{h} 43^{m} 42^{\mathrm{s}} .3$ and $3^{h} 44^{m} 56$ s 6 , and $\operatorname{Dec}(\mathrm{J} 2000)+31^{\circ} 56^{\prime} 00^{\prime \prime}$ and $\left.+32^{\circ} 19^{\prime} 52^{\prime \prime}\right)$ in the 1-0 S(1) line of molecular hydrogen at $2.122 \mu \mathrm{m}$. Outflow activity was only detected towards the south-western limit of the cluster, in the region of the well-known HH 211 outflow (see Fig. 2). This region coincides with the area in which Strom et al. (1974) had found the embedded infrared source IC 348-IR and Bachiller, Guilloteau \& Kahane (1987) found several clumps in ammonia and CO. These signs show that star formation is still going on in this part of the IC 348 cluster.

Apart from $\mathrm{HH} 211$, we find $\mathrm{H}_{2}$ emission features spreading over five regions near HH 211, which are outlined in Fig. 2. These regions labelled 1 to 5 are shown enlarged as contour plots in Figs. 3 to 6 . In these plots, the more prominent $\mathrm{H}_{2}$ emission features have been labelled, and their positions and fluxes are given in Table1. Also marked are possible outflow sources in the field: the "+" signs show the positions of $3.5 \mathrm{~cm}$ sources detected by Avila et al. (2001).

The most prominent of these new $\mathrm{H}_{2}$ features is a large, roughly north-south oriented outflow. Its brightest parts could already be seen on the $\mathrm{K}^{\prime}$ image of Mc Caughrean et al. (1994), where it

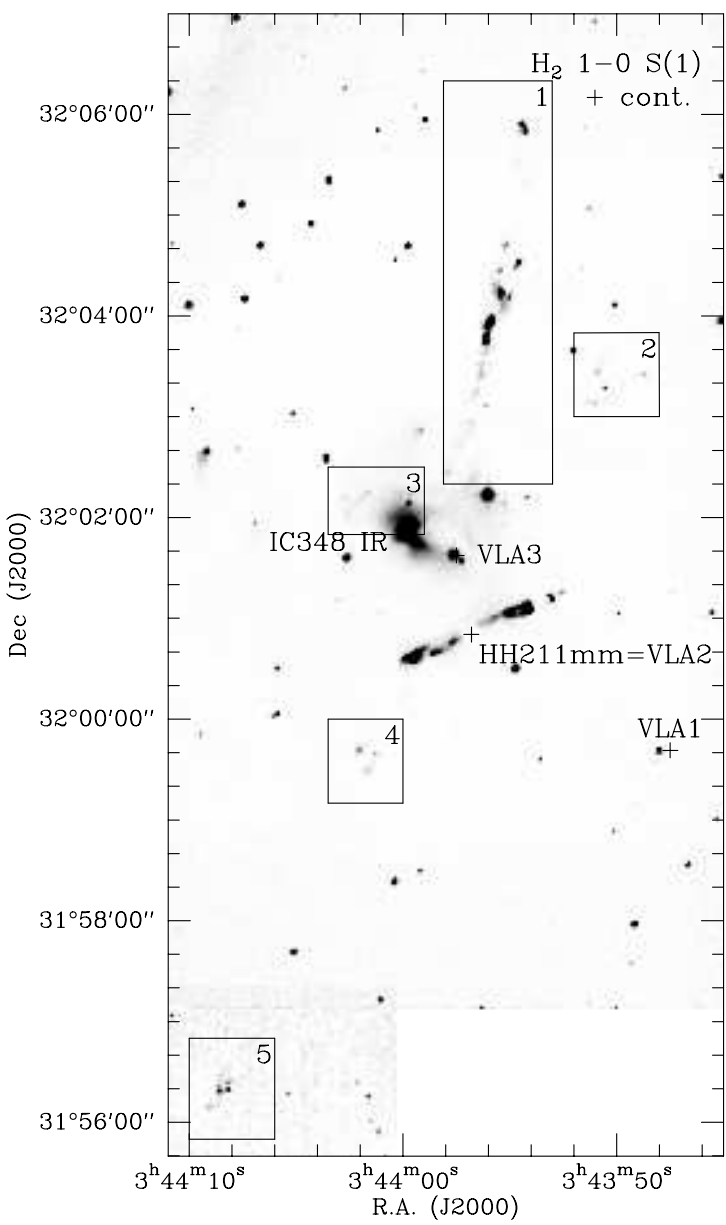

Fig. 2.- HH211 and the newly discovered $\mathrm{H}_{2}$ emission knots at $2.122 \mu \mathrm{m}$. Boxes mark the regions which are displayed in detail as contour plots. The + signs mark the $3.5 \mathrm{~cm}$ sources detected by Avila et al. (2001).

was referred to as the "chain" of knots. Its northern part is shown in Fig. 3. It consists of a strand of knots, almost 4 arcmin long, which splits up in the region between knots 1-1 and 1-s. Here, we are probably seeing condensations that are part of a large bow shock. After a gap of about $30^{\prime \prime}$, a few more $\mathrm{H}_{2}$ knots are seen, which end in the bowshaped knots $1-\mathrm{v} / \mathrm{w}$. The flow shows a clearly visible bending, of about $10^{\circ}$ towards the east, over its length of almost $4^{\prime}$. Such bending is also observed in several other jets and outflows (see, e.g. Eislöffel (2000)). A variety of possible explanations have been given for such bending by Fendt and Zinnecker (1998), Terquem et al. (1999), and 


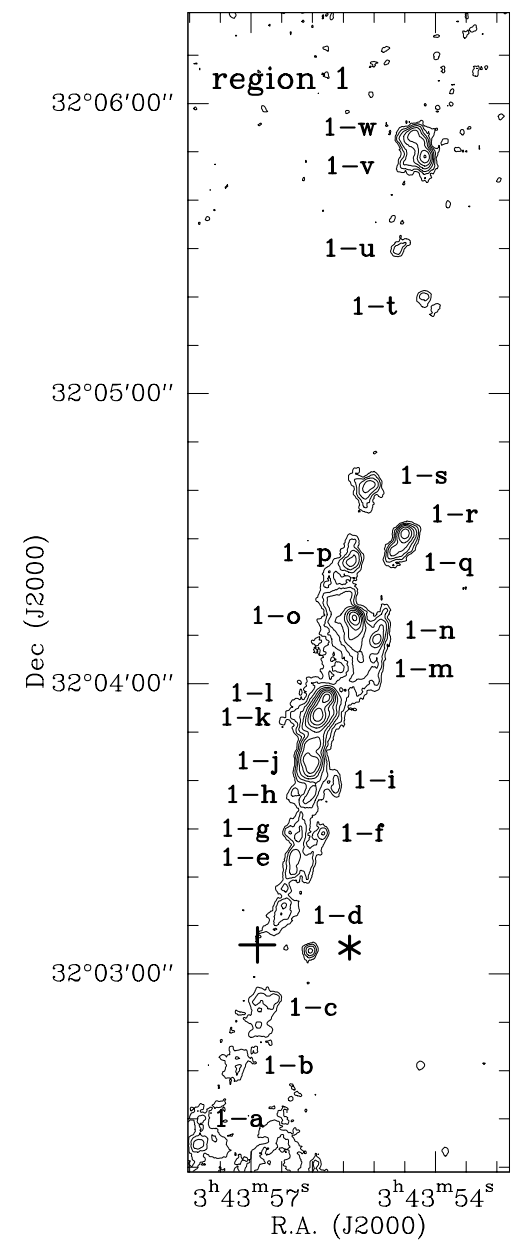

Fig. 3.- Contour plot of region 1 at $2.122 \mu \mathrm{m}$. The contours start at the $1.5 \times 10^{-19} \mathrm{Wm}^{-2} \operatorname{arcsec}^{-2}$ and then increase by a factor of $\sqrt{3}$. The "+" sign marks the position of IC $348 \mathrm{MMS}$, while the "*" means the object is not a $\mathrm{H}_{2}$ emission feature but a star.

Eislöffel (2000): Lorentz forces on a magnetic jet, motion of the source in a binary system, warping of the disk or dynamical pressure of the external medium. Here, we do not yet have enough information about the kinematics of the flow's knots available that would permit us to distinguish between these possibilities.

South of HH 211, in regions 4 and 5 (see Fig. 4), we find two more compact groups of $\mathrm{H}_{2}$ knots, which we interpret as southward pointing bow shocks. These bows have positions and orientations which suggest that they belong to the southern lobe of the large north-south outflow. They are not aligned on a straight line with the north-
Table 1: Positions and fluxes of newly found $\mathrm{H}_{2}$ knots. Positional errors are on the order of $0{ }^{\prime \prime} 5$. Photometric errors range from $5 \%$ for the brighter and up to $20 \%$ for the fainter knots.

\begin{tabular}{|c|c|c|c|c|}
\hline Object & $\alpha(\mathrm{J} 2000)$ & $\delta(\mathrm{J} 2000)$ & Flux* & $\begin{array}{c}\text { Surface** } \\
\text { Brightness }\end{array}$ \\
\hline $1-\mathrm{a}$ & $03: 43: 57.8$ & $+32: 02: 25$ & 14.3 & 7.2 \\
\hline $1-b$ & $03: 43: 57.2$ & $+32: 02: 43$ & 10.2 & 3.1 \\
\hline $1-\mathrm{c}$ & 03:43:56.6 & $+32: 02: 56$ & 18.3 & 5.0 \\
\hline $1-\mathrm{d}$ & $03: 43: 56.5$ & $+32: 03: 14$ & 17.3 & 5.4 \\
\hline $1-\mathrm{e}$ & $03: 43: 56.3$ & $+32: 03: 25$ & 21.9 & 7.0 \\
\hline $1-\mathrm{f}$ & $03: 43: 55.9$ & $+32: 03: 30$ & 4.7 & 5.5 \\
\hline $1-\mathrm{g}$ & $03: 43: 56.3$ & $+32: 03: 30$ & 11.8 & 6.7 \\
\hline $1-\mathrm{h}$ & 03:43:56.1 & $+32: 03: 38$ & 13.4 & 7.1 \\
\hline $1-\mathrm{i}$ & $03: 43: 55.7$ & $+32: 03: 40$ & 6.0 & 4.3 \\
\hline $1-j$ & $03: 43: 56.1$ & $+32: 03: 44$ & 91.0 & 41.0 \\
\hline $1-\mathrm{k}$ & $03: 43: 56.0$ & $+32: 03: 54$ & & 120.6 \\
\hline $1-1$ & $03: 43: 55.8$ & $+32: 03: 58$ & 99.4 & 101.5 \\
\hline $1-\mathrm{m}$ & $03: 43: 55.3$ & $+32: 04: 05$ & 30.9 & 9.3 \\
\hline $1-n$ & $03: 43: 55.0$ & $+32: 04: 10$ & 23.8 & 24.2 \\
\hline $1-\mathrm{o}$ & $03: 43: 55.4$ & $+32: 04: 15$ & 114.0 & 92.5 \\
\hline $1-p$ & $03: 43: 55.4$ & $+32: 04: 27$ & 17.8 & 11.6 \\
\hline $1-q$ & $03: 43: 54.8$ & $+32: 04: 28$ & 393 & 12.9 \\
\hline $1-r$ & $03: 43: 54.6$ & $+32: 04: 32$ & 50.5 & 40.3 \\
\hline $1-\mathrm{s}$ & $03: 43: 55.2$ & $+32: 04: 42$ & 19.8 & 12.2 \\
\hline $1-\mathrm{t}$ & $03: 43: 54.3$ & $+32: 05: 21$ & 6.5 & 4.2 \\
\hline $1-u$ & $03: 43: 54.7$ & $+32: 05: 31$ & 6.6 & 3.9 \\
\hline $1-\mathrm{v}$ & $03: 43: 54.3$ & $+32: 05: 50$ & 71.9 & 47.7 \\
\hline $1-\mathrm{w}$ & $03: 43: 54.5$ & $+32: 05: 54$ & 11.9 & 22.5 \\
\hline $2-\mathrm{a}$ & 03:43:51.1 & $+32: 03: 08$ & 10 & 4.8 \\
\hline $2-\mathrm{b}$ & $03: 43: 50.9$ & $+32: 03: 08$ & 10.2 & 7.0 \\
\hline $2-\mathrm{c}$ & $03: 43: 50.5$ & $+32: 03: 09$ & 4.0 & 3.2 \\
\hline $2-d$ & $03: 43: 50.1$ & $+32: 03: 12$ & 2.5 & 3.2 \\
\hline $2-\mathrm{e}$ & $03: 43: 48.9$ & $+32: 03: 25$ & 2.8 & 3.8 \\
\hline $2-f$ & $03: 43: 48.6$ & $+32: 03: 25$ & 6.4 & 12.1 \\
\hline $2-\mathrm{g}$ & $03: 43: 50.8$ & $+32: 03: 26$ & 11.8 & 7.8 \\
\hline $2-\mathrm{h}$ & $03: 43: 50.7$ & $+32: 03: 33$ & 6.9 & 3.1 \\
\hline $3-\mathrm{a}$ & $03: 44: 02.6$ & $+32: 02: 11$ & 1.1 & 5.4 \\
\hline $3-b$ & $03: 44: 02.5$ & $+32: 02: 00$ & 3.5 & 4.4 \\
\hline $3-\mathrm{c}$ & 03:44:02.0 & $+32: 02: 08$ & 2.8 & 3.6 \\
\hline $3-d$ & $03: 44: 01.7$ & $+32: 02: 12$ & 48 & 5.5 \\
\hline $3-\mathrm{e}$ & $03: 44: 01.5$ & $+32: 02: 14$ & 4.8 & 7.1 \\
\hline $3-f$ & $03: 43: 59.6$ & $+32: 02: 08$ & 11.5 & 30.4 \\
\hline 4-a & $03: 44: 01.4$ & $+31: 59: 27$ & 14.8 & 5.3 \\
\hline $4-b$ & $03: 44: 01.8$ & $+31: 59: 40$ & 20.8 & 9.2 \\
\hline $5-\mathrm{a}$ & $03: 44: 08.7$ & $+31: 56: 05$ & 5.2 & 4.1 \\
\hline $5-b$ & $03: 44: 08.2$ & $+31: 56: 15$ & 10.8 & 7.6 \\
\hline $5-\mathrm{c}$ & 03:44:07.8 & $+31: 56: 16$ & 6.4 & 10.2 \\
\hline $5-d$ & 03:44:07.8 & $+31: 56: 20$ & 5.2 & 4.3 \\
\hline
\end{tabular}

* Fluxes are in $10^{-18} \mathrm{~W} \mathrm{~m}^{-2}$ summed over the whole knot.

** Surface brightness of $\mathrm{H}_{2} \quad 1-0 \mathrm{~S}(1)$ averaged over a $1^{\prime \prime}$ diameter are in $10^{-19} \mathrm{~W} \mathrm{~m}^{-2} \operatorname{arcsec}^{-2}$. 

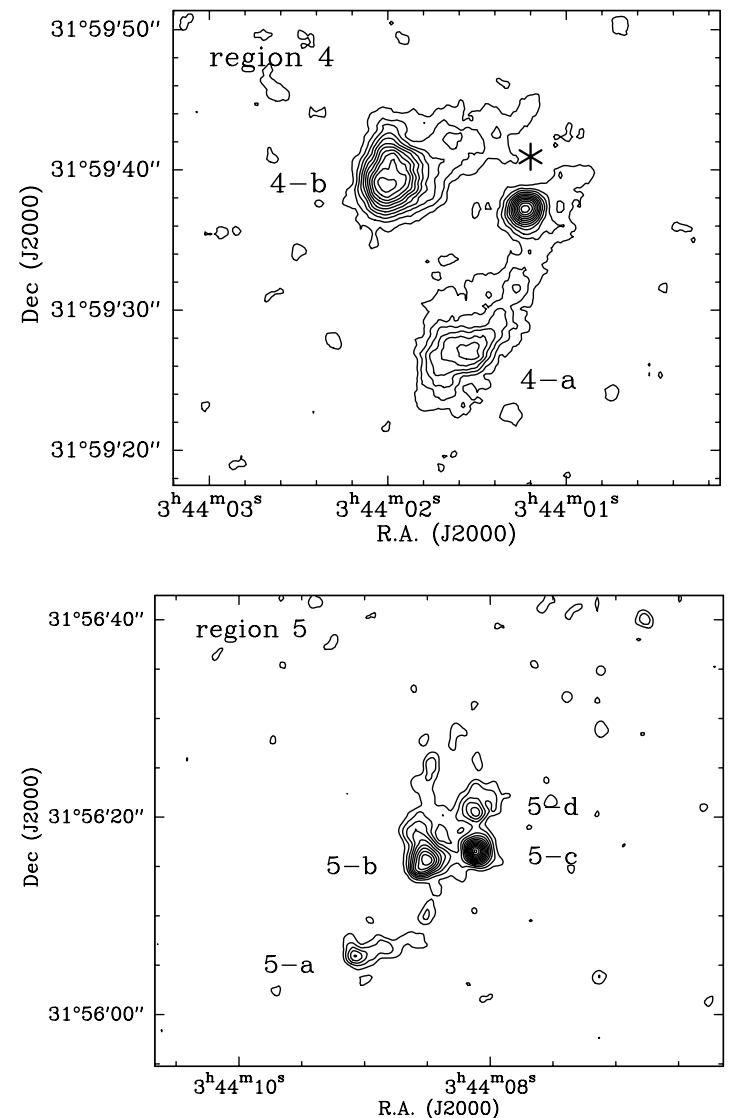

Fig. 4.- Contour plot of region 4 (upper panel) and region 5 (lower panel) at $2.122 \mu \mathrm{m}$. The contours start at $1.5 \times 10^{-19} \mathrm{Wm}^{-2} \operatorname{arcsec}^{-2}$ in both panels, but increase by a factor of $\sqrt{3}$ in the upper panel and linearly by $7.5 \times 10^{-20} \mathrm{Wm}^{-2} \operatorname{arcsec}^{-2}$. The "*" marks a star.

ern flow, but instead also show a clear bending of the flow towards the east in the southern lobe. The total length of this flow would then be $10^{\prime}$ or $0.87 \mathrm{pc}$ at a distance of $300 \mathrm{pc}$. We note, that in projection this flow passes very close by the southeastern bow of $\mathrm{HH} 211$. Although we do not detect $\mathrm{H}_{2}$ emission from it in this region, observations of HH 211 in other tracers may do so: In their interferometric study of $\mathrm{CO}$ in the $\mathrm{HH} 211$ flow, Gueth and Guilloteau (1999) found CO emission at $8.2 \mathrm{~km} \mathrm{~s}^{-1}$ in this region, which was hard to explain if it came from HH 211 itself. We suggest that this emission may not belong to the HH 211 outflow, but instead comes from the large northsouth flow.

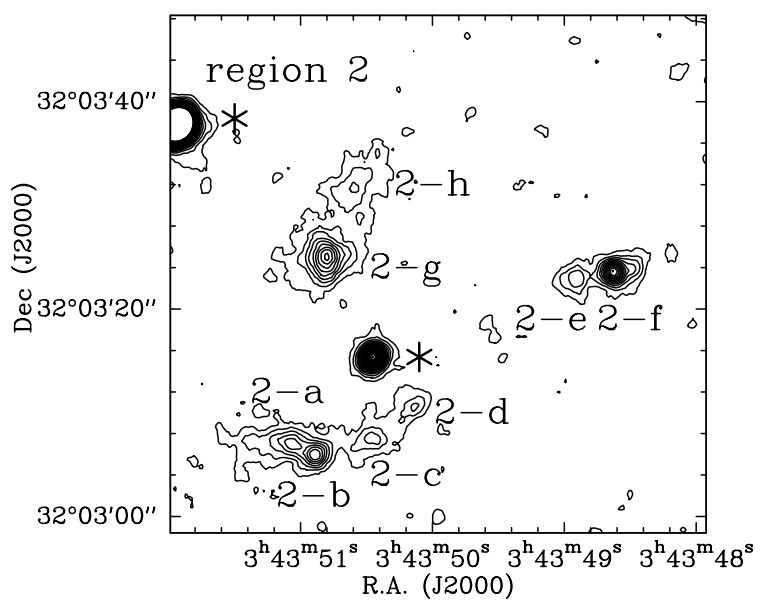

Fig. 5.- Contour plot of region 2 at $2.122 \mu \mathrm{m}$. The contours start at the $1 \times 10^{-19} \mathrm{Wm}^{-2} \operatorname{arcsec}^{-2}$ level and increase linearly in steps of $7.5 \times 10^{-20} \mathrm{Wm}^{-2} \operatorname{arcsec}^{-2}$. The "*" sign means the object is a star, not a $\mathrm{H}_{2}$ emission feature.

In addition to the large north-south flow, we find two more regions of $\mathrm{H}_{2}$ emission north of HH 211. These regions 2 and 3 are shown in detail in Figs. 5 and 6. Both consist of compact groups of knots, which again resemble incomplete bow shocks, that may be pointing south-east (region 2 ) and east (region 3). Because of their orientations, we speculate that these objects do not belong to the same outflow. We note, however, that the bows in regions 2 and 5 are on a line that passes very close by the HH 211 source. Therefore, it cannot be excluded that they form a flow emanating from the HH 211 source as well (which then should be a binary).

For the new north-south outflow we measure a total flux in the $1-0 \mathrm{~S}(1)$ line of $\mathrm{H}_{2}$ of $6.8 \times 10^{-16} \mathrm{Wm}^{-2}$. This converts to a luminosity of about $1.9 \times 10^{-3} \mathrm{~L}_{\odot}$ in this line. From this value we can roughly infer the intrinsic total $\mathrm{H}_{2}$ luminosity of this flow: In order to estimate the flux in all $\mathrm{H}_{2}$ lines, we assume about $10 \%$ to be emitted in the 1-0 S(1) line alone (which is the case, e.g., for C-shocks of $30-40 \mathrm{kms}^{-1}$ shock speed, typical for such flows (Smith 1991). With an average extinction of $\mathrm{A}_{K} \approx 2 \mathrm{mag}$ for this region of the young IC 348 cluster (see Mc Caughrean et al. (1994)), we find a total instrinsic $\mathrm{H}_{2}$ luminosity for the flux in region 1 of $0.1 \mathrm{~L}_{\odot}$. For the 


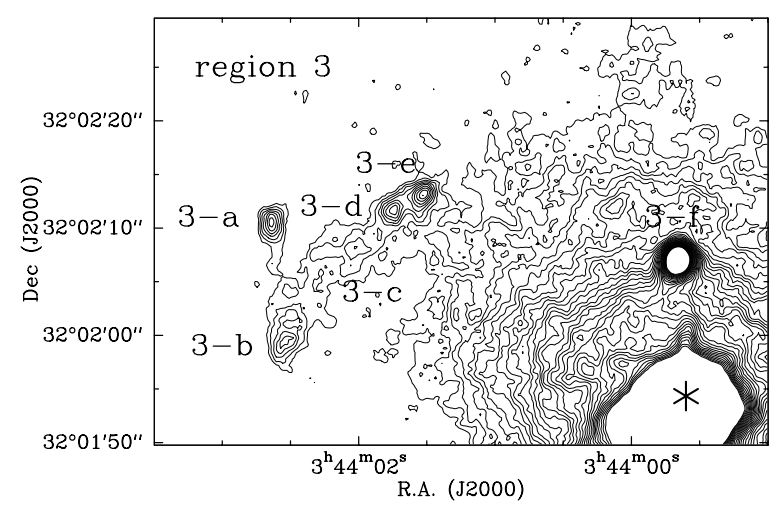

Fig. 6. - Contour plot of region 3 at $2.122 \mu \mathrm{m}$. The contours start at the $1.8 \times 10^{-19} \mathrm{Wm}^{-2} \operatorname{arcsec}^{-2}$ level and increase linearly in steps of $5 \times 10^{-20} \mathrm{Wm}^{-2} \operatorname{arcsec}^{-2}$. The "*" marks IC348 IR .

other regions we measure the following fluxes in the $1-0 \mathrm{~S}(1)$ line of $\mathrm{H}_{2} 3.3 \times 10^{-17} \mathrm{Wm}^{-2}$ (region 2 ), $1.6 \times 10^{-17} \mathrm{Wm}^{-2}$ (region 3 ), $3.6 \times 10^{-17}$ $\mathrm{Wm}^{-2}$ (region 4) and $3.8 \times 10^{-17} \mathrm{~W} \mathrm{~m}^{-2}$ (region 5 ), and estimate the intrinsic total $\mathrm{H}_{2}$ luminosity in the same way as $4.7,2.3,5.1,5.4 \times 10^{-3} \mathrm{~L}_{\odot}$, respectively. Due to overlap effects the total flux for a region may slightly differ from a sum of the respective knots as given in Table 1.

We also searched for morphological and photometric variability in the $\mathrm{H}_{2}$ knots. Such variability should be expected due to the short cooling times in $\mathrm{H}_{2}$ of order one year for the shock excited gas in the outflows. Nonetheless, we find at most very small or no changes at all in the brightness of the knots over the five year timespan that our $\mathrm{H}_{2}$ observations cover.

Since from morphology alone it is difficult to assign the newly found molecular emission features to individual outflows, we tried to determine preliminary proper motions for the knots. Due to the coarse pixel scale, especially of our earliest observations, the small number of available epochs and the usable epoch difference of only 3.2 years, we currently reach a measurement accuracy of only $300 \mathrm{~km} \mathrm{~s}^{-1}$. None of our measured knots is found to move that fast. This is not unexpected, since in most flows for which precise proper motions could be determined, values of around $200 \mathrm{~km} \mathrm{~s}^{-1}$ were found (see, e.g., Eislöffel and Mundt (1992), Eislöffel and Mundt (1994), Micono et al. (1998)).

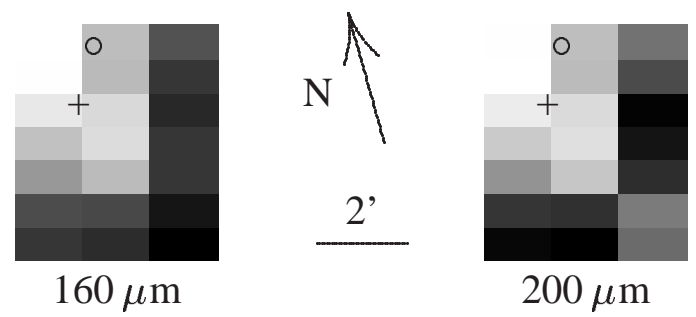

Fig. $\quad$ 7. - ISOPHOT maps at $160 \mu \mathrm{m}$ and $200 \mu \mathrm{m}$ centered on $\mathrm{HH} 211-\mathrm{mm}$. Also marked are IC 348 IR (plus) and IC 348 MMS (circle). Bright sources are white and sky background is black.

\section{Outflow sources and star formation in IC 348}

In a survey of the region at $3.5 \mathrm{~cm}$, Avila et al. (2001) found three VLA sources, where VLA2 is identical with HH 211-mm, while another variable source VLA3 was found very close to the star $\mathrm{Cl}^{*}$ IC348 LRL49. They suggested that this star may be the driving source of the north-south outflow.

In order to search for possible exciting sources of the new molecular outflow features, we obtained maps with ISOPHOT at $160 \mu \mathrm{m}$ and $200 \mu \mathrm{m}$, as well as mm-continuum data with the MAMBO bolometer array at $1.2 \mathrm{~mm}$. Our ISOPHOT maps (Fig. 7) were centred on HH 211-mm. Strong emission from this source was detected at both wavelengths. It is, however, affected by emission from other point or extended sources to the north and north-east, which are not resolved at the resolution of ISO. Our mm-map (Fig. 8), by contrast, clearly resolves point sources and extended emission.

The most prominent source in the field is HH 211-mm. There appears to be a previously unknown, fainter point-like source to the south-west. This second source is also indicated as a small elongation of HH 211-mm in the submillimetre maps of Chandler and Richer (2000), especially at $450 \mu \mathrm{m}$ and $750 \mu \mathrm{m}$. It was not, however, detected by Avila et al. (2001) at $7 \mathrm{~mm}$ and $3.5 \mathrm{~cm}$. The available data do not yet allow us to discern whether this object is a secondary to HH 211-mm or only a dust condensation at the edge of the outflow cavity.

Both point sources are embedded in a halo 


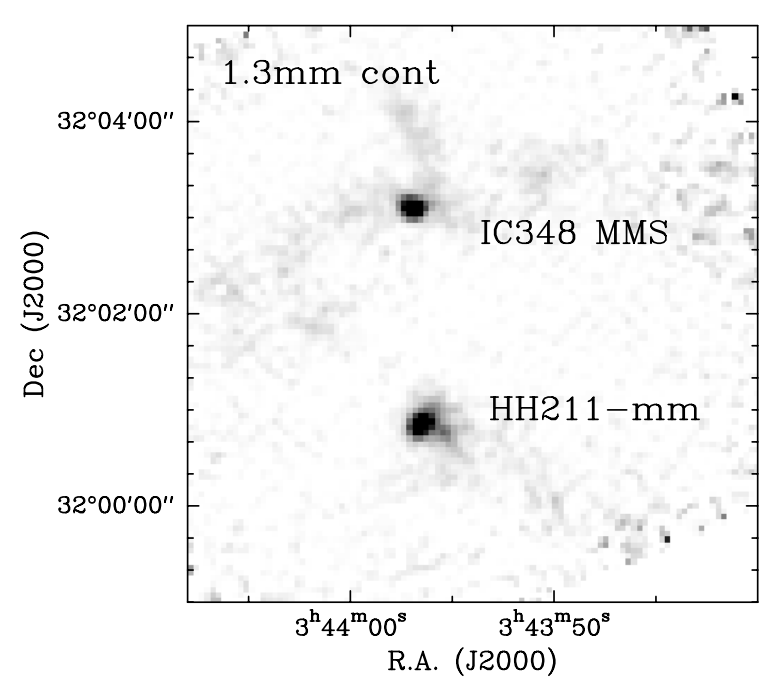

Fig. 8.- MAMBO $1.3 \mathrm{~mm}$ map showing the HH 211-mm (south) and the newly discovered IC 348 MMS source (north).

of diffuse emission, which extends further to the south-west to the edge of our map. This "dust ridge" coincides with the $\mathrm{H}^{13} \mathrm{CO}^{+}$filament seen by Gueth and Guilloteau (1999). In a 30" aperture centred on HH 211-mm (at a position of $3^{h} 43^{m} 56.5,+32^{\circ} 00^{\prime} 51^{\prime \prime}(\mathrm{J} 2000)$ ), we measure a total flux of $1.88 \mathrm{Jy}$, of which $0.89 \mathrm{Jy}$ come from the HH 211-mm point source itself, while the rest has to be attributed to the diffuse halo and the "companion".

About 2 arcmin north of HH 211-mm we find a hitherto unknown mm-source, which we call IC 348 MMS. This source is also detected in the ISOPHOT maps, where its position (at $3^{h} 43^{m} 56^{\mathrm{s}} 9,+32^{\circ} 03^{\prime} 06^{\prime \prime}(\mathrm{J} 2000)$ ) has been marked with a circle. It is situated on a saddle point on an emission ridge between two of the $\mathrm{NH}_{3}(1,1)$ cores observed by Bachiller, Guilloteau \& Kahane (1987). This ridge and the cores are also seen as faint dust filaments on our continuum map. We measure a flux of $1.58 \mathrm{Jy}$ in a $30^{\prime \prime}$ aperture for this source.

Since IC 348 MMS is situated in the gap between the $\mathrm{H}_{2}$ knots 1-c and 1-d (see Fig. 3), we suggest it to be the driving source of the long north-south molecular outflow. Measurements of the radial velocities of the $\mathrm{H}_{2}$ knots to its north and south should confirm that this object and not VLA3 drives the long outflow. The fact that this source is bright at far-infrared and mmwavelengths, but is not seen at all in the nearinfrared makes it likely that it is in the Class 0 stage of its evolution, similar to HH 211-mm: in our ISOPHOT maps we measure a flux of 55.3 and $48.2 \mathrm{Jy}$ at $160 \mu \mathrm{m}$ and $200 \mu \mathrm{m}$, respectively, for IC 348 MMS (see Froebrich et al. (2003) for details of how such fluxes are measured in ISOPHOT maps). Together with the flux of $1.58 \mathrm{Jy}$ at $1.2 \mathrm{~mm}$ given above we can construct an initial spectral energy distribution, which will allow us a comparison with the SEDs of other known Class 0 sources (especially HH 211-mm) and will give us hints about the nature of this object. By fitting a modified black body to these measurements we derive a bolometric temperature as defined by Myers and Ladd (1993) of $\mathrm{T}_{b o l}=43 \mathrm{~K}$, a luminosity of $\mathrm{L}_{b o l}=8 \mathrm{~L}_{\odot}$, a power-law exponent of the frequency dependence of the dust opacity of $\beta=0.7$, and a $\mathrm{L}_{s m m} / \mathrm{L}_{b o l}=0.026$. This ratio would classify the source as a Class 0 object, if an extended protostellar envelope is found (see André et al. (2000)). Taking the evolutionary model of Froebrich et al. (2003) we can further derive an age estimate of $29000 \mathrm{yr}$, a current stellar mass of $0.1 \mathrm{M}_{\odot}$, a final stellar mass of $0.5 \mathrm{M}_{\odot}$, and an envelope mass of $1.1 \mathrm{M}_{\odot}$ for IC $348 \mathrm{MMS}$. These calculations assume a dust opacity of $4 \mathrm{~cm}^{2} \mathrm{~g}^{-1}$ at $12 \mu \mathrm{m}$ and follow the approach of Myers et al. (1998). The observed $1.2 \mathrm{~mm}$ flux ratio of HH 211$\mathrm{mm}$ to IC $348 \mathrm{MMS}$ of 1.2 is in good agreement with the modelled envelope mass ratio of 1.3 (see also Froebrich et al. (2003)). Thus IC 348 MMS seems to be well in the range of parameters for a Class 0 source and may be a bit more evolved than HH 211-mm, although because of the extended dust seen in the ISOPHOT maps and its position close to the edge of the maps the errors are large. Follow-up with SIRTF is clearly necessary to derive the parameters with higher precision.

As a result of our $\mathrm{H}_{2}$ survey, we conclude that at the south-western edge of IC 348 star formation is still active. In this region a small subcluster of embedded young stellar objects is currently forming. No signs of outflow activity or equally young stars are found in other parts of IC 348. At least some of the subcluster members (HH 211-mm, IC 348 MMS, IC 348 IR) are in such early stages of their evolution (the Class 0 
and Class 1 phases) that they are probably much younger than the youngest of the optically visible objects investigated by Herbig (1998). The unusually large age-spread of the IC 348 cluster members that Herbig found may then be understood in terms of a formation scenario of this cluster, in which subclusters similar to the one seen here have formed at various times (and possibly various places) within the cluster. Since the crossing time in IC 348 is on the order of $0.5 \mathrm{Myr}$, older subgroups would, however, have been spread all over the cluster in the meantime, and should no longer appear as localized clumps of similar age. The exception appears to be the subcluster discussed in this paper, which is so young that its members have not yet moved far from their birthplaces.

\section{Conclusions}

We have carried out a wide-field $\mathrm{H}_{2}$ survey of the embedded young open cluster IC 348. Outflow activity was only found at its south-western limit, in the region of HH 211. Here, a small subcluster of very deeply embedded obejcts still in the very early phases of their evolution seems to be forming. The considerable age-spread in IC 348 observed by Herbig (1998) could be understood if IC 348 had been built up from such subclusters that formed at different times.

In this part of IC 348, we observe a large northsouth oriented outflow, and identify a newly discovered mm-object IC $348 \mathrm{MMS}$ as its source. For several other groups of $\mathrm{H}_{2}$ knots, on the other hand, we cannot identify their sources.

$\mathrm{HH} 211-\mathrm{mm}$ is resolved as a double point-like source in our mm-map, although it is not yet clear if the "secondary" is a physical companion or a dust condensation in the outflow cavity wall. Both HH 211-mm and IC 348 MMS are also detected at $160 \mu \mathrm{m}$ and $200 \mu \mathrm{m}$ with ISOPHOT. Both sources probably are going through the Class 0 phase of their evolution.

Our deep images in the 1-0 S(1) line of molecular hydrogen trace the HH 211 jet and counterjet as highly-collimated straight chains of knots. Both resemble the molecular jets observed in interferometric maps in $\mathrm{CO}$ and $\mathrm{SiO}$. This jet system appears to be rotated about $3^{\circ}$ counter-clockwise with respect to the system of the prominent $\mathrm{H}_{2}$ bow shocks. We interpret the CO emission ahead of the south-eastern bow observed by Gueth and Guilloteau (1999) - and difficult to understand if it was associated with the HH 211 outflow - as belonging to the IC 348 MMS outflow, which is crossing the HH 211 flow in projection in that region.

We thank Manfred Stickel for his help with the data reduction of the ISOPHOT data, and Pavel Kroupa for an interesting discussion on sequential cluster formation. An anonymous referee has helped to clarify this paper. Jochen Eislöffel and Dirk Froebrich received financial support from DLR through Verbundforschung grant 50 OR 99049, Mark J. McCaughrean through grant 50 OR 0004. The ISOPHOT data presented in this paper were reduced using PIA, which is a joint development by the ESA Astrophysics Division and the ISOPHOT Consortium with the collaboration of the Infrared Processing and Analysis Center (IPAC). Contributing ISOPHOT Consortium institutes are DIAS, RAL, AIP, MPIK and MPIA.

\section{REFERENCES}

André, P., Ward-Thompson, D., Barsony, M. 2000, Protostars and Planets IV, V. Mannings, A.P. Boss, S.S. Russell (eds.) p.59

Avila, R., Rodríguez, L.F., Curiel, S. 2001, Revista Mexicana de Astronomía y Astrofísica, 37, 201

Bachiller, R., Guilloteau, S., Kahane, C. 1987, A\&A, 173, 324

Bizenberger, P., McCaughrean, M.J., Birk, C., Thompson, D., Storz, C. 1998, SPIE, 3354, 825

Boulard, M.-H., Caux, E., Monin, J.-L., Nadeau, D., Rowlands, N. 1995, A\&A, 300, 276

Cernicharo, J., Bachiller, R., Duvert, G. 1985, A\&A, 149, 273

Chandler, C.J., Richer, J.S. 2000, ApJ, 530, 851

Chandler, C.J., Richer, J.S. 2001, ApJ, 555, 139

Eislöffel, J. 2000, A\&A, 354, 236

Eislöffel, J., Mundt, R. 1992, A\&A, 263, 292 
Eislöffel, J., Mundt, R. 1992, A\&A, 284, 530

Fendt, C., Zinnecker, H., 1998, A\&A, 334, 750

Froebrich, D., Smith, M.D., Hodapp, K.-W., Eislöffel, J., 2003, submitted

Gueth, F., Guilloteau, S. 1999, A\&A, 343, 571

Herbig, G.H. 1998, AJ, 497, 736

Herbst, T.M., Birk, C., Beckwith, S.V.W., Hippler, S., Mc Caughrean, M.J., Mannucci, F., Wolf, J. 1993, Proc. SPIE, 1946, 605

Kreysa, E., Gemuend, H.-P., Gromke, J., et al. 1998, Proc. SPIE, 3357, 319

Lada, E.A., Lada, C.J. 1995, AJ, 109, 1682

Luhman, K.L., Rieke, G.H., Lada, C.J., Lada, E.A. 1998, ApJ, 508, 347

Mc Caughrean, M.J., Rayner, J.T., Zinnecker, H. 1994, AJ, 436, L189

Myers, P.C., Ladd, E.F. 1993, ApJ, 413, L47

Myers, P.C., Adams, F.C., Chen, H., Schaff, E. 1998, ApJ, 492, 703

Micono, M., Davis, C.J., Ray, T.P., Eislöffel, J., Shetrone, M.D. 1998, ApJ494, L227

Najita, J.R., Tiede, G.P., Carr, J.S. 2000, ApJ, 541,977

Preibisch, T., Zinnecker, H., Herbig, G.H. 1996, A\&A, 310, 456

Preibisch, T., Zinnecker, H. 2001, AJ, 122, 866

Rengel, M., Froebrich, D., Hodapp, K., Eislöffel, J. 2002, in: The Origins of Stars and Planets: The VLT View, João Alves \& Mark McCaughrean (ed.)

Rengel, M., Froebrich, D., Hodapp, K., Eislöffel, J. 2001, in: R. E. Schielicke (Hrsg.): Astron. Ges. Abstract Ser. 18 (2001), 168

Smith, M.D. 1991, MNRAS, 253, 175

Strom, S.E., Strom, K.M., Carrasco, L. 1974, PASP, 86, 798

Terquem, C., Eislöffel, J., Papaloizou, J.C.B., Nelson, R.P. 1999, ApJ512, L131

This 2-column preprint was prepared with the AAS LATEX

macros v5.0. 